\title{
ARTICLE
}

\section{Anomalous hall effect in a triangular-lattice antiferromagnet $\mathrm{UNi}_{4} \mathrm{~B}$}

\author{
Akira Oyamada $^{\mathrm{a}^{*}}$, Takao Inohara ${ }^{\mathrm{a}}$, Etsuji Yamamoto ${ }^{\mathrm{b}}$ and Yoshinori Haga ${ }^{\mathrm{b}}$ \\ ${ }^{a}$ Graduate School of Human and Environmental Studies, Kyoto University, Kyoto, 606-8501, Japan; ${ }^{b} J a p a n$ Atomic Energy Agency, \\ 2-4 Shirakata, Tokai-mura, Naka-gun, Ibaraki-ken, 319-1195, Japan
}

\begin{abstract}
We report the Hall effect measurements on a triangular-lattice antiferromagnet $\mathrm{UNi}_{4} \mathrm{~B}$ in the context of the proposed relevance of the ferroic toroidal order in its magnetically ordered state below $T_{\mathrm{N}}=20 \mathrm{~K}$. It has been proposed theoretically that the ferroic toroidal order offers a new route to the electromagnetic effect. One of the most remarkable phenomena originated from the electromagnetic effects is the Hall voltage without magnetic field. Our measurements show that the Hall voltage is proportional to the square of the electric current and its coefficient increases significantly below $T_{\mathrm{N}}$, which supports the theoretical prediction.
\end{abstract}

Keywords: magnetoelectric effect; ferroic toroidal order; anomalous Hall effect; uranium compound; $\mathrm{UNi}_{4} \mathrm{~B} ;$ frustration; triangular lattice;

\section{Introduction}

The toroidal moments in condensed-matter physics have attracted much attention recently since the ferroic ordering of the toroidal moments may offer a new-type magnetoelectric effect [1]. Several compounds show the ferroic toroidal order, however, all of them are insulators so far [2-4]. A triangular-lattice antiferromagnet $\mathrm{UNi}_{4} \mathrm{~B}$ is a promising metallic candidate that has the ferroic order of the toroidal moments.

The ferroic toroidal order in $\mathrm{UNi}_{4} \mathrm{~B}$ is realized as the distinctive magnetic structure on the triangular lattice as shown in Figure 1. Neutron diffraction measurements showed that the magnetic structure of $\mathrm{UNi}_{4} \mathrm{~B}$ below $T_{\mathrm{N}}=$ $20 \mathrm{~K}$ is a vortex-like arrangement of two-thirds of uranium electronic spins (blue arrows in the Figure 1) and one-third of them remained paramagnetic (blue filled circles) [5]. If we only see the ordered spins, the spins form a honeycomb lattice. In the magnetically ordered state, the toroidal moment is expressed as

$$
\boldsymbol{t}=\frac{g \mu_{B}}{2} \sum_{i} \boldsymbol{r}_{i} \times \boldsymbol{S}_{i}
$$

where $g$ is the $g$ factor, $\mu_{\mathrm{B}}$ is the Bohr magneton, $\boldsymbol{r}_{\mathrm{i}}$ is the each position of spins and $S_{\mathrm{i}}$ is the localized spin. According to Eq.(1), each vortex-like structure made of six arrows around a shaded area in Figure 1 corresponds to one toroidal moment. The direction of the toroidal moment is perpendicular to the page. As the direction of the all vortices in Figure 1 are the same, the magnetic structure is regarded as the ferroic toroidal order [6,7].

*Corresponding author. Email:oyamada.akira.2s@kyoto-u.ac.jp

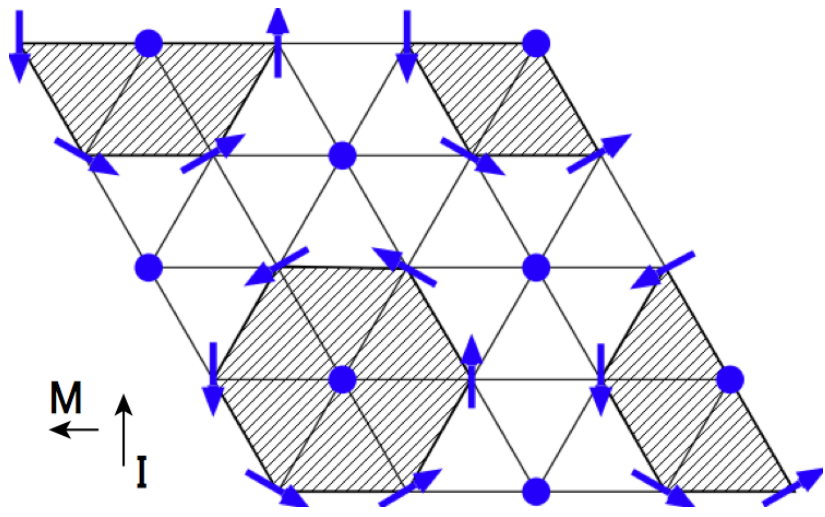

Figure 1. Magnetic Structure of $\mathrm{UNi}_{4} \mathrm{~B}$ below $T_{\mathrm{N}}=20 \mathrm{~K}$. The blue arrows show the ordered spins and the blue filled circles show the paramagnetic spins. Each vortex-like structure made of six arrows around a shaded area corresponds to one toroidal moment. The direction of the electric current (I) for the Hall measurements and the predicted direction of induced magnetization $(\mathrm{M})$ are indicated.

The theoretical investigations by Hayami et al. demonstrated that the ferroic toroidal order in $\mathrm{UNi}_{4} \mathrm{~B}$ is relevant to originate the magnetoelectric effects. They constructed the low-energy effective single-band Hubbard-type model with a site-dependent antisymmetric spin-orbit coupling. This model gives the magnetization induced by the electric current, the highly anisotropic Hall responses and the modulation of the electronic band structure [6]. Their discussions are based on the geometrical condition; ordered spins on a honeycomb lattice, the existence of the global space-inversion symmetry and the breaking of the local space-inversion symmetry. The crystal structure of 
$\mathrm{UNi}_{4} \mathrm{~B}$ is $\mathrm{C}$-centered orthorhombic [7]. The geometrical condition of the theory is approximately satisfied though the hexagonal structure shown in Figure 1 is slightly distorted.

In order to investigate the ferroic toroidal order specifically in $\mathrm{UNi}_{4} \mathrm{~B}$, the following two points were taken into account [8]. The first one is the model Hamiltonian. They used the extended Anderson model with the antisymmetric spin-orbit coupling that is suitable for f-electron systems. The second one is the realistic crystal structure. The crystal structure is layered triangular lattice and there are two kinds of paramagnetic sites surrounded by $\mathrm{Ni}$ atoms or $\mathrm{B}$ atoms. They took the above points into account. This investigation revealed that the specific model for $\mathrm{UNi}_{4} \mathrm{~B}$ gives several ordered phases. Among them, there is a metallic vortex-like ordered phase, which is the same as that of $\mathrm{UNi}_{4} \mathrm{~B}$. This ordered phase shows almost the same electromagnetic effects as the effective single-band Hubbard-type model [8]. Actually the origin of the vortex-like magnetic structure is still controversial. This theory offers a promising explanation of the magnetic structure.

Among the proposed interesting effects, here we note the Hall voltage without magnetic field induced by the ferroic toroidal order. It was proposed that when the electric current $(I)$ flows in the triangular-lattice plane (TL plane), a uniform magnetization is induced perpendicular to $I$ in the TL plane as shown in Figure 1. This induced magnetization originates the current along the axis perpendicular to the TL plane (TL axis) and the Hall voltage is induced along the TL axis (please see Figure 2). The voltage is proportional to $I^{2}$ in contrast to the usual case. This is a kind of anomalous Hall effect without spontaneous magnetization. In this paper we report the temperature dependence of the Hall voltage without magnetic field and try to confirm the theoretical prediction.

\section{Experimental method}

As mentioned later, the Hall voltage under zero magnetic field is so small that the detection of the voltage is not easy. Figure 2 shows the schematic view of the electric contacts on a single crystal. In the figure $V+$ and $V$ - are the voltage contacts and the $I+$ and $I$ - are the current contacts. The rectangle is the single-crystal sample. The sample dimension is $1.9 \mathrm{~mm} \times 0.7 \mathrm{~mm} \times 0.3$ $\mathrm{mm}$. The single-crystal sample was prepared so that the current flows in the TL plane and the Hall voltage is measured along the TL axis. There is an inevitable deviation $\Delta L$ between two voltage contacts. If $\Delta L$ is not zero, the additional voltage between $V+$ and $V$ - is originated from the resistivity. The additional voltage may mask the Hall voltage. In order to decrease the additional voltage we applied the following procedure. We made two current contacts and one voltage contact at first. Applying current through the current contacts and measuring the voltage through the voltage contacts, we made the last voltage contact using a manipulator so that the voltage originated by the deviation $\Delta L$ becomes as small as possible. The contact resistance was about 1 $\mathrm{Ohm}$ between the electric contacts and $0.5 \mathrm{Ohm}$ between the voltage contacts.

The current was supplied by a regulated current source and the voltage was measured by a nano-volt meter.

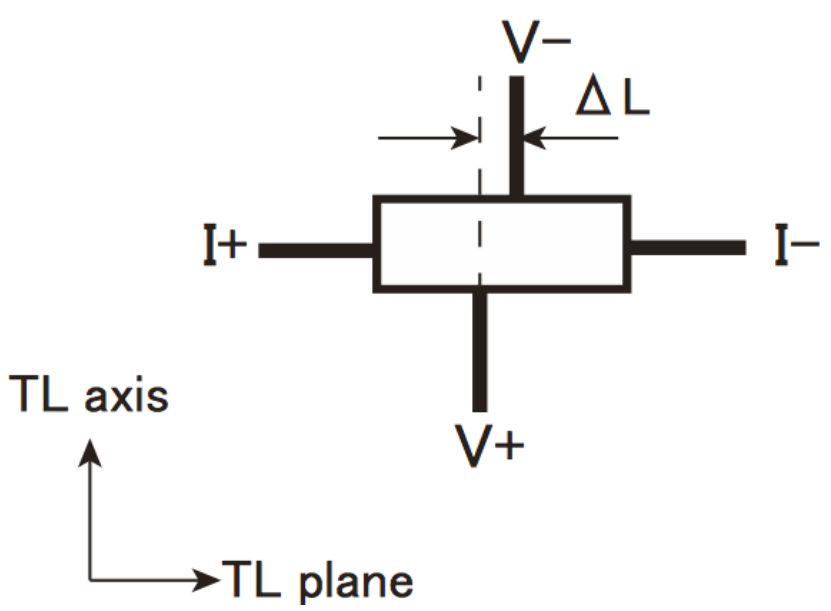

Figure 2. Schematic view of the electric contacts on the sample. The rectangle is the single-crystal sample. $\Delta L$ is the deviation between the positions of $V+$ and $V$-. The size of $\Delta L$ in the figure is exaggerated.

\section{Results and discussions}

The Hall voltage $\left(V_{\mathrm{H}}\right)$ as a function of the electric current $I$ was measured using a single crystal of $\mathrm{UNi}_{4} \mathrm{~B}$ as shown in Figure 3. A red symbol shows the $V_{\mathrm{H}}$ curve above $T_{\mathrm{N}}$ and blue symbols show the $V_{\mathrm{H}}$ below $T_{\mathrm{N}}$. The current $I$ was applied in the TL plane and $V_{\mathrm{H}}$ was measured along the TL axis. $V_{\mathrm{H}}$ has a large I-linear contribution and a rather small $I^{2}$ contribution. The small change of $V_{\mathrm{H}}$ is observable below $T_{\mathrm{N}}$, while there is almost no temperature dependence on $V_{\mathrm{H}}$ above $T_{\mathrm{N}}$.

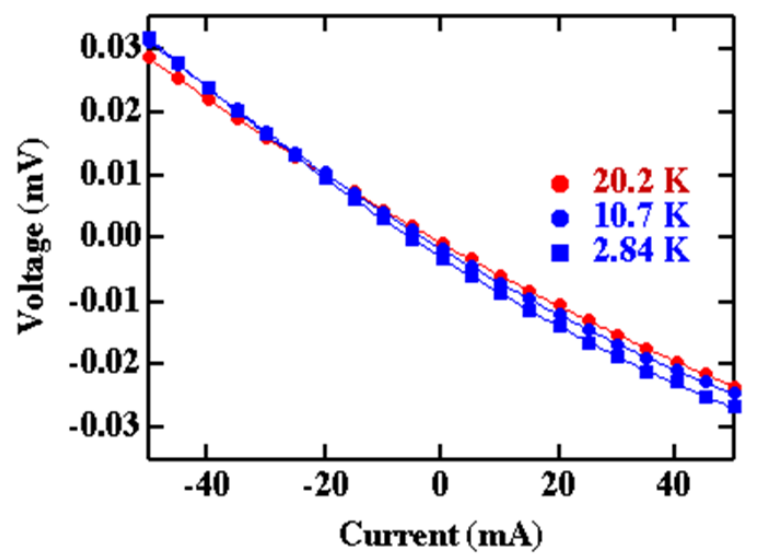

Figure 3. Hall voltage as a function of the electric current. There is a small change below $T_{\mathrm{N}}$. Red filled circles show the $V_{\mathrm{H}}$ curve above $T_{\mathrm{N}}$ and blue filled circles and squares show the $V_{\mathrm{H}}$ below $T_{\mathrm{N}}$. 
The $V_{\mathrm{H}}$ vs $I$ curve is well described by the equation $V_{\mathrm{H}}=\mathrm{C}_{0}+\mathrm{C}_{1} I+\mathrm{C}_{2} I^{2}$ at each temperature. The fitting range for the applied current was between 0 and $50 \mathrm{~mA}$. Here we examined the each parameter. The parameter $\mathrm{C}_{0}$ is the zero-point shift of the nanovoltmeter. As mentioned in section 2, the small deviation between the voltage contacts gives the additional voltage originated by the resistivity in the TL plane. The temperature dependence of the fitting parameter $\mathrm{C}_{1}$ should be consistent with that of the resistivity in the TL plane. We measured the resistivity in the TL plane to confirm the consistency. The temperature dependences of the parameter $\mathrm{C}_{1}$ and the resistivity in the TL plane are shown in Figure 4. In the figure, $C_{1}$ is multiplied by the factor as mentioned below. At first we compare the resistivity with the previous result. It has already reported that the resistivity has a large anisotropy $[9,10]$. The resistivity along the TL axis has small minimum around $100 \mathrm{~K}$ and slightly increases down to $T_{\mathrm{N}}$. There is a sharp decrease below $T_{\mathrm{N}}$, that is apparently different from the TL-plane resistivity. The resistivity in the TL plane increases monotonously with decreasing temperature and there is no anomaly at $T_{\mathrm{N}}$. The resistivity we measured in the TL plane is qualitatively the same as the previous result. Secondly we compare the resistivity we measured and the parameter $C_{1}$. The temperature dependence of both is almost the same as shown in Figure 4. The multiplied factor of the $C_{1}$ corresponds to the deviation $\Delta L=0.06 \mathrm{~mm}$, which seems to be reasonable. Thus we conclude that the parameter $C_{1}$ is originated by the resistivity in the TL plane.

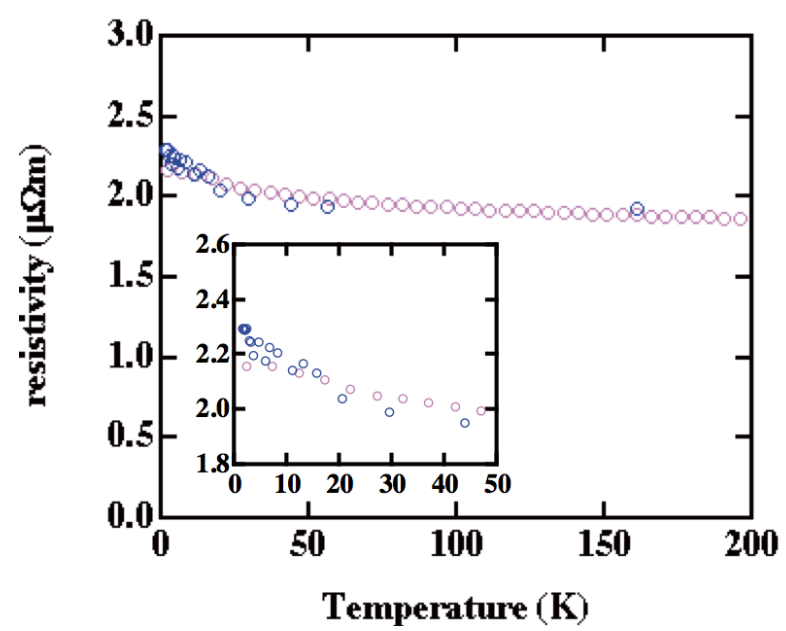

Figure 4. Temperature dependence of the resistivity (pink circles) and the fitting parameter $\mathrm{C}_{1}$ (blue circles) of the Hall voltage (blue circles). The parameter $\mathrm{C}_{1}$ in the figure is multiplied by the factor, which is explained in the text.

Actually, there is a small difference between reference 9 and reference 10 . The resistivity in the TL plane has a significant decrease $(4 \%$ of the total resistivity) below $5 \mathrm{~K}$ in reference 10 but not in reference 9. We measured the resistivity several times and there was no decrease below $5 \mathrm{~K}$. This difference may be due to the sample dependence or sample alignment and require further studies.

Figure 5 shows the temperature dependence of the $I^{2}$ coefficient $\mathrm{C}_{2}$. The coefficient $\mathrm{C}_{2}$ is constant above $T_{\mathrm{N}}$, while there is a remarkable increase below $T_{\mathrm{N}}$. The temperature dependence of $\mathrm{C}_{2}$ below $T_{\mathrm{N}}$ is just like an order parameter suggesting that the increase of $\mathrm{C}_{2}$ is associated with the ferroic toroidal order. The value of $\mathrm{C}_{2}$ at $2 \mathrm{~K}$ is about $2.1 \times 10^{-3} \mathrm{~V} / \mathrm{A}^{2}$. When $10 \mathrm{~mA}$ is applied, the value corresponds to $0.2 \mu \mathrm{V}$ that is close to the limit of the measurement accuracy. We conclude that the increase of $\mathrm{C}_{2}$ below $T_{\mathrm{N}}$ is significant. However the quantitative discussion of the temperature dependence of $\mathrm{C}_{2}$ is difficult.

Now we examine the increase of $\mathrm{C}_{2}$ below $T_{\mathrm{N}}$. In usual case, the Hall voltage is proportional to the electric current and the magnetic field. The application of the positive and negative magnetic field cancels out the additional voltage due to $\Delta L$. In our measurements this cancelling could not be carried out because the magnetic field was not applied. Instead, the fitting of the $V_{\mathrm{H}}$ curve can distinguish between the additional voltage and the Hall voltage since the Hall voltage is proportional to $I^{2}$ in our measurements. On the other hand, the application of the positive and negative current cancels the thermoelectric voltage originated by the temperature gradient in the sample in usual case. In our measurements, this cancelling could not be carried out because the Hall voltage was also cancelled. Therefore the measured $\mathrm{C}_{2}$ possibly includes the contribution of the thermoelectric voltage. The temperature dependence of the thermoelectric power (TEP) for $\mathrm{UNi}_{4} \mathrm{~B}$ has already reported [10]. The TEP is highly anisotropic. The TEP in the TL plane has a small value between room temperature and $2 \mathrm{~K}$ and approaches zero at the lowest temperature. The TEP along the TL axis shows a wide maximum around $230 \mathrm{~K}$ and decrease with decreasing temperature. Furthermore it has a kink at $T_{\mathrm{N}}$ and approaches zero below $T_{\mathrm{N}}$. Even if the large temperature gradient occurs in the sample, the increase

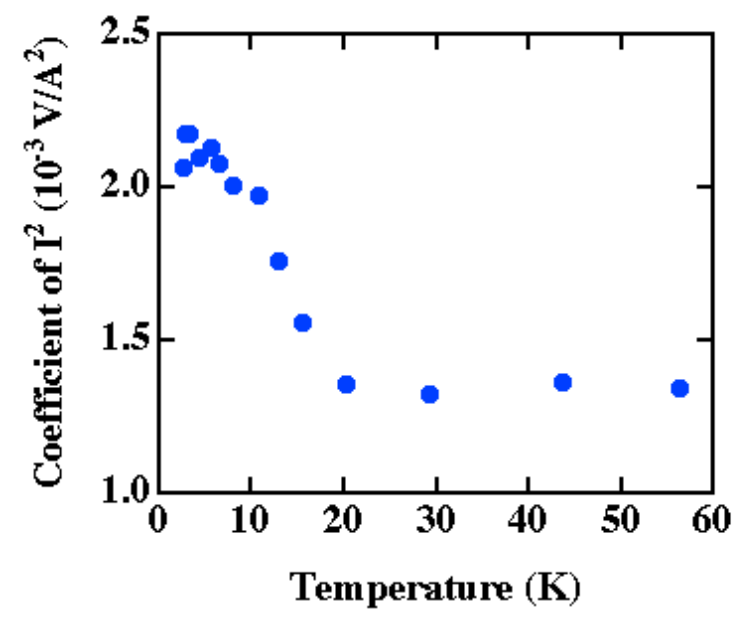

Figure 5. Temperature dependence of the fitting parameter $\mathrm{C}_{2}$ of the Hall voltage. The increase of $\mathrm{C}_{2}$ below $T_{\mathrm{N}}$ is significant. 
of the $\mathrm{C}_{2}$ is not explained since the TEP decreases below $T_{\mathrm{N}}$.

We turn to the constant value $\mathrm{C}_{2}$ above $T_{\mathrm{N}}$. The theory predicted the Hall voltage without magnetic field is zero although we observed that $\mathrm{C}_{2}$ is constant up to $160 \mathrm{~K}$. One possibility is the thermoelectric voltage in the TL plane. The voltage contacts are along the TL axis. However the voltage induced by the TEP in the TL plane is possibly observed because of the deviation $\Delta L$. When the electric current is applied between the current contacts, the heating occurs mainly at the two electric contacts on the sample since the contact resistances are much bigger than the sample resistance. Then the temperature gradient in the sample is brought about by the difference between the values of the two contact resistances. As the temperature dependence of the TEP in the TL plane is small, the $\mathrm{C}_{2}$ above $T_{\mathrm{N}}$ seems to be explained by the voltage induced by the TEP qualitatively. On the contrary, the quantitative explanation is difficult. Our result shows that the Hall voltage from the $\mathrm{C}_{2}$ contribution is about $5 \mu \mathrm{V}$ when the $50 \mathrm{~mA}$ is applied. As the TEP in the TL plane is roughly $1 \mu \mathrm{V} / \mathrm{K}$, the temperature difference between the voltage contacts should be $5 \mathrm{~K}$, which is not plausible. Therefore the origin of the $\mathrm{C}_{2}$ above $T_{\mathrm{N}}$ is unclear for the moment.

Now we comment on the problem of domains. The magnetic ordering has domains in general. In the case of $\mathrm{UNi}_{4} \mathrm{~B}$, each vortex structure of spins has the same direction in one domain but can be opposite in another domain. This situation leads to the toroidal domains. If the total area of domains with the clockwise vortex and that with the counterclockwise vortex is the same, the contribution to the electromagnetic effect from the all domains canceled out. Thus our observation indicates that there is a difference between the both areas. We tried to control the domains by cooling the sample with the application of electric current along the TL axis several times without success. Nevertheless the data were reproducible. The problem of domains requires further studies.

\section{Conclusion}

The anomalous Hall effect without magnetic field was studied. We observed that the Hall voltage has the contribution that is proportional to the square of the applied electric current. This contribution increases significantly below $T_{\mathrm{N}}$ suggesting the existence of the electromagnetic effect originated from the ferroic toroidal order. The results support the theoretical prediction.

\section{Acknowledgements}

The authors wish to thank S. Hayami, H. Kusunose and Y. Motome for fruitful discussions.

\section{References}

[1] N.A. Spaldin, M. Fiebig and M. Mostovoy, The toroidal moment in condensed-matter physics and its relation to the magnetoelectric effect, J. Phys.: Condens. Matter. 20 (2008), pp.434203/1-15.

[2] T-H Arima, J-H Jung, M Matsubara, M Kubota, J-P He, Y. Kaneko and Y. Tokura, Resonant Magnetoelectric X-ray Scattering in $\mathrm{GaFeO}_{3}$ : Observation of Ordering of Toroidal Moments, $J$. Phys. Soc. Jpn., 74 (2005), pp.1419-1422.

[3] A.S. Zimmermann, D. Meier and M. Fiebig, Ferroic nature of magnetic toroidal order, Nat. Commun. 5 (2014), pp.4796/1-5.

[4] P. Toledano, M. Ackermann, L. Bohaty, P. Becker, T. Lorenz, N. Leo and M. Fiebig, Primary ferrotoroidicity in antiferromagnets, Phys. Rev. B 92 (2015) pp.094431/1-7.

[5] S.A.M. Mentink, A. Drost, G.J. Nieuwenhuys, E. Frikkee, A.A. Menovsky and J.A. Mydosh, Magnetic ordering and frustration in hexagonal $\mathrm{UNi}_{4} \mathrm{~B}$, Phys. Rev. Lett., 73 (1994), pp.1031-1034.

[6] S. Hayami, H. Kusunose and Y. Motome, Toroidal order in metals without local inversion symmetry, Phys. Rev. B 90 (2014), pp.024432/1-12.

[7] Y. Haga, A. Oyamada, T.D. Matsuda, S. Ikeda and Y. Onuki, Crystal structure of frustrated antiferromagnet $\mathrm{UNi}_{4} \mathrm{~B}$, Physica B403 (2008) pp.900-902.

[8] S. Hayami, H. Kusunose and Y. Motome, Toroidal order in a partially disordered state on a layered triangular lattice: implication to $\mathrm{UNi}_{4} \mathrm{~B}, J$. Phys.: Conf. Series. 592 (2015), pp.012101/1-6.

[9] S.A.M. Mentink, H. Nakotte, A.de Visser, A.A. Menovsky, G.J. Nieuwenhuys and J.A. Mydosh, Reduced-moment antiferromagnetism in single-crystal $\mathrm{UNi}_{4} \mathrm{~B}$, Physica B186-188 (1993) pp. 270-272.

[10]S.A.M. Mentink, G.J. Nieuwenhuys, A.A. Menovsky, J.A. Mydosh, A. Drost and E. Frikkee, Antiferromagnetic order and spin frustration in $\mathrm{UNi}_{4} \mathrm{~B}$, Physica B 206\&207 (1995) pp.473-475.

[11]Y. Bando, T. Suemitsu, K. Takagi, H. Tokushima, Y. Echizen, K. Katoh, K. Umeo, Y. Maeda and T. Takabatake, Large thermoelectric power in several metallic compounds of cerium and uranium, $J$. Alloys Comp. 313 (2000) pp.1-6. 